\title{
Perfil de pacientes hipertensos em uma drogaria de Porto Alegre-RS
}

\author{
Hypertensive profile of patients from a drugstore in Porto Alegre-RS
}

\author{
Editiane Alcantara PETRUZZELLIS, \\ Fernanda Maciel RODRIGUES, Patrícia Martins BOCK \\ Centro Universitário Metodista - IPA, Rua Coronel Joaquim Pedro Salgado, 80 - \\ Rio Branco, Porto Alegre - RS, 90420-060. \\ E-mail: patriciabock74@gmail.com
}

\section{ABSTRACT}

We conducted a quantitative, cross-sectional study. Data were collected through semi-structured interviews, guided by script previously tested. The following aspects were evaluated: patient profile, knowledge about hypertension, treatment and drug-related problems, and checking blood pressure of each participant. The sample consisted of 97 patients. Patients presenting hypertension family history constituted $91.8 \%$ of the sample. It was found that $57.7 \%$ were overweight, $56.7 \%$ did not adopt salt restriction and $73.2 \%$ had no physical activity. Only $45.4 \%$ are careful about the time to take the medications, $69.1 \%$ do not follow doctor frequently, and the difficulty of consulting was the main reason, mentioned by $42.3 \%$. It was observed that $44.3 \%$ is not aware of the consequences of hypertension and $27.8 \%$ did not know about the chronicity of the disease. Drug treatment of $58.8 \%$ was mono-therapy. Since that with pharmaceutical care patients can have better responses to treatment of hypertension, the results of poor adherence to treatment observed in this study allowed us to critical analysis and reflective of the need to enhance the pharmaceutical care of this drugstore, and explain users the benefits and risks of their antihypertensive medications, in order to lead them to a healthier lifestyle, eliminate or control the risks of hypertension.

Keywords: hypertension; medication adherence; pharmaceutical care

\section{RESUMO}

Foi realizado um estudo quantitativo, observacional transversal. Os dados foram obtidos por meio de entrevista semiestruturada, orientada por roteiro previamente testado. Foram avaliadas: características do paciente, conhecimento sobre hipertensão arterial, tratamento e problemas relacionados aos medicamentos, e foi medida pressão arterial de cada participante. A amostra foi constituída de 97 pacientes. Os indivíduos com familiares que apresentavam hipertensão constituíram $91,8 \%$ da amostra. Verificou-se que 57,7\% estavam com sobrepeso, 56,7\% não adotava restrição de sal e $73,2 \%$ não fazia atividades físicas de nenhum tipo. Apenas $45,4 \%$ são cuidadosos quanto ao horário de uso dos medicamentos, $69,1 \%$ não faz acompanhamento médico freqüentemente, sendo a dificuldade de conseguir consulta o motivo principal, apontado por $42,3 \%$. Observou-se que $44,3 \%$ não tem conhecimento das conseqüências da hipertensão e $27,8 \%$ não sabem sobre a cronicidade da doença. O tratamento medicamentoso de $58,8 \%$ foi a monoterapia. Uma vez que com a atenção farmacêutica pode-se ter melhores respostas ao tratamento da hipertensão arterial sistêmica, os resultados de baixa adesão ao tratamento observados neste estudo nos possibilitaram uma análise crítica e reflexiva sobre a necessidade de intensificar o atendimento farmacêutico desta drogaria, e explicar aos usuários os benefícios e os riscos de seus medicamentos anti-hipertensivos, com a finalidade de conduzi-los a um estilo de vida mais saudável, eliminar ou controlar os riscos da HAS.

Palavras Chave: hipertensão; adesão a medicação; atenção farmacêutica 


\section{INTRODUÇÃO}

A hipertensão arterial sistêmica (HAS) caracterizase pela continuidade dos níveis da pressão arterial (PA) elevados. A linha demarcatória que define a HAS considera valores de pressão arterial sistólica (PAS) $\geq$ $140 \mathrm{mmHg}$ e de pressão arterial diastólica (PAD) $\geq 90$ mmHg (1). A HAS tem alta prevalência e baixas taxas de controle, o diagnóstico deverá ser sempre validado por medidas repetidas, em condições ideais, em, pelo menos, três ocasiões em consultório médico. Após sua confirmação, deve ser classificada como hipertensão primária, secundária, terciária e isolada, além da verificação do prejuízo dos órgãos alvos e levantamento de outros fatores de risco cardiovasculares $(2,3)$.

A HAS é uma doença crônica, geralmente assintomática. Sua evolução clínica é lenta, com uma multiplicidade de fatores causais. Quando não tratada adequadamente, traz graves complicações, temporárias ou permanentes $(4,5)$. A HAS pode causar provavéis danos adicionais, além da simples expansão do volume intravascular. A PA elevada crônica leva à lesão vascular, e lesões do coração, rins e cérebro são decorrentes das lesões vasculares desses órgãos. Devido a esses efeitos sobre orgãos alvo, ela é considerada um importante fator de risco que predispõe as doenças cardiovasculares, cerebrovasculares e insuficiência renal crônica; por este motivo, é responsável pela alta morbidade e mortalidade dos pacientes (6). Sendo a HAS uma doença crônica, pode ser controlada, mas não curada, requerendo tratamento por toda a vida (7).

Atingir a PA ótima é o objetivo mais importante para os pacientes hipertensos $(8,9)$. O tratamento da HAS é baseado em recursos não-farmacológico e farmacológico, sendo que a adesão do paciente ao tratamento é imprescindível. $\mathrm{O}$ tratamento não-farmacológico presta-se ao controle dos fatores de risco e às modificações no estilo de vida, a fim de prevenir ou deter a evolução da HAS (10). A terapia medicamentosa, apesar de eficaz na redução dos valores pressóricos, da morbidade e da mortalidade, tem alto custo e pode ter efeitos colaterais, motivando o abandono do tratamento (9). Qualquer que seja a opção, é fundamental obter a adesão continuada dos pacientes às medidas recomendadas para a obtenção de um controle adequado da PA (11).

Para a escolha da terapia medicamentosa adequada, deve se levar em consideração o tipo de hipertensão e qualquer outra condição clínica que o paciente possa apresentar (9). As classes de anti-hipertensivos disponíveis para uso clínico são: diuréticos (medicamentos de $1^{\circ}$ escolha para o tratamento da HAS, devido a evidências científicas comprovadas em estudos clínicos comparativos com outras classes, e relação custo-benefício favorá- vel), inibidores adrenérgicos (dividos em agonistas alfa 2 de ação central, betabloqueadores e alfabloqueadores), vasodilatadores diretos, bloqueadores dos canais de cálcio, inibidores da enzima conversora da angiotensina (IECA) e bloqueadores do receptor AT1 da angiotensina II (ARA II). Cada uma das diferentes classes de fármacos anti-hipertensivos possui propriedades específicas, com vantagens e desvantagens. As associações de anti-hipertensivos podem ser feitas por meio de monofármacos ou associações de princípios ativos $(1,3)$.

Mesmo sendo passível de controle por meio de tratamentos medicamentosos, os índices de controle da HAS ainda são baixos. O controle insatisfatório da doença tem relação direta com a baixa adesão ao tratamento, sendo este um impedimento direto para o alcance dos objetivos terapêuticos (11). Acredita-se que a informação correta e de fácil compreensão possa auxiliar na adesão do paciente ao tratamento (12). O paciente portador de HAS, consequentemente, através da atenção farmacêutica, terá uma melhor adesão ao seu tratamento, tendo assim ótimos resultados no controle de sua doença (13). Com mais conhecimento e menos dúvidas em relação a sua doença, o paciente se sente mais confiante e seguro para dar continuidade à terapia medicamentosa, o que evita complicações futuras decorrentes da doença e melhora a qualidade de vida (14).

Este trabalho teve por objetivo descrever o perfil de pacientes hipertensos em uma drogaria de Porto Alegre-RS, bem como o conhecimento desses pacientes sobre a hipertensão arterial, buscando descobrir quais são seus principais erros em relação ao tratamento medicamentoso e estilo de vida, para que através dos resultados deste estudo a atenção farmacêutica desta drogaria possa ser intensificada, auxiliando assim no correto tratamento anti-hipertensivo.

\section{MATERIAL E MÉTODOS}

Esta pesquisa se constituiu de um estudo observacional transversal, com análise de dados quantitativos. O estudo foi desenvolvido na Drogaria Compre Bem, localizada zona leste da cidade de Porto Alegre, Estado do Rio Grande do Sul. Esta região é caracterizada por ter uma população de renda baixa a média, desta forma a escolha desta drogaria permite que os dados coletados reflitam uma população que utiliza principalmente o sistema único de saúde para tratamento médico, e foi escolhida para que os resultados do presente estudo possam posteriormente ser utilizados como subsídios para a ampliação das práticas de atenção farmacêutica.

Para este trabalho foram avaliados 97 indivíduos, para ser obtido um nível de confiança de $95 \%$ e um erro máximo de $10 \%$. Foi realizada amostragem aleatória e 
os critérios de inclusão foram: pacientes atendidos em uma Drogaria comercial; de ambos os sexos; com idade igual ou superior a 18 anos; que apresentassem diagnóstico médico de hipertensão arterial, que fizessem uso de qualquer tratamento anti-hipertensivo e aceitassem participar da pesquisa. Foram excluídas da pesquisa gestantes.

Os dados foram obtidos por meio de formulário estruturado, no período de fevereiro a abril de 2012, previamente testado. $\mathrm{O}$ instrumento foi elaborado a partir de estudos originais que continham questões que avaliassem a adesão e conhecimento quanto ao tratamento de doenças diversas. Durante a fase de testagem o instrumento de pesquisa foi avaliado por meio de sua aplicação em sete indivíduos, avaliação da interpretação de cada item do instrumento, de forma a desenvolver um questionário facilmente compreendido por indivíduos de diferentes níveis socioculturais.

Os dados foram organizados em categorias posteriormente determinadas e analisadas com base nas experiências dos entrevistados frente à doença e ao tratamento. As categorias levantadas foram: características do paciente, apreciação do seu conhecimento sobre hipertensão arterial, tratamento e problemas relacionados aos medicamentos, juntamente com a observação da verificação da pressão arterial de cada participante. A verificação da pressão arterial foi realizada antes do questionário na sala de procedimentos da Drogaria com o paciente sentado, com o braço direito apoiado ao nível do coração, depois de quinze minutos de descanso, para normalização da pressão arterial. A verificação foi realizada com aparelho digital de pulso da marca More Sitness (MF-368), previamente calibrado, cedido pela Drogaria Compre Bem.

As variáveis pesquisadas foram: gênero, idade, escolaridade, renda familiar, conhecimento da cronicidade da HA, conhecimento das consequências da HA, se familiares também possuem HA, anti-hipertensivos em uso, informações do estilo de vida do paciente: se fuma, se pratica atividades físicas ou ingere bebidas alcoólicas; informações em relação à alimentação: se ingere verduras, frutas, alimentos gordurosos; se apresenta restrições ao sal; informações em relação ao tratamento: melhora com o tratamento, adesão ao tratamento, acompanhamento do tratamento com profissionais da saúde, relação com os profissionais da saúde, antecedentes de doenças cardiovasculares e valor da $\mathrm{PA} \mathrm{em} \mathrm{mm} / \mathrm{Hg}$.

$\mathrm{O}$ projeto de pesquisa foi aprovado pelo Comitê de Ética em Pesquisa do Centro Universitário Metodista do IPA sob o $n^{\circ}$ de aprovação 267/2011. A coleta de dados foi precedida da Autorização do proprietário da Drogaria Compre Bem, juntamente com o Termo de Consentimento Livre e Esclarecido assinado pelos entrevistados. Aos participantes foi assegurado o anonimato e o direito de retirar o consentimento no momento que desejassem.

Os dados foram organizados em um banco de dados e foi realizada análise estatística descritiva, com apresentação dos resultados em freqüências e média e desvio padrão. O programa utilizado para a análise foi o software Statistical Package for Social Sciences (SPSS versão 17.0).

\section{RESULTADOS}

Este estudo avaliou 97 indivíduos, sendo a maior parte do gênero feminino (58,8\%). Na amostra 36,1\% dos pacientes pertenciam a faixa etária entre 30 a 45 anos, $37,1 \%$ entre 45 a 60 anos e $26,8 \%$ com idades superiores a 60 anos.

$\mathrm{Na}$ Tabela 1 pode ser visualizado o perfil destes pacientes. O nível de instrução prevalente desta população foi o ensino fundamental $(51,5 \%)$, e a renda familiar entre 3 e 4 salários mínimos $(51,5 \%)$. Os indivíduos com familiares que apresentavam hipertensão constituíram 91,8\% da amostra, sendo que a maior parte desta população nunca apresentou nenhuma das doenças vasculares citadas $(95,9 \%)$. Verificou-se que a maior parte da população da amostra $(57,7 \%)$ encontrava-se com sobrepeso. Em relação à alimentação os pacientes responderam que algumas vezes ingeriam alimentos gordurosos $(91,8 \%)$, frequentemente frutas $(69,1 \%)$ e verduras $(62,9 \%)$. Mais da metade $(56,7 \%)$ não apresentavam restrição de sal e $25,8 \%$ ingeriam bebidas alcoólicas. Mais da metade não faziam atividades físicas $(73,2 \%)$ e uma pequena parte destes indivíduos faziam uso de tabaco $(22,7 \%)$.

As respostas relacionadas com a adesão, uso do serviço de saúde e o conhecimento do paciente ao tratamento anti-hipertensivo, encontram-se descritas na Tabela 2. Verificou-se que somente 18,6\% dos pacientes lembravam-se de tomar seus medicamentos, $45,4 \%$ são cuidadosos quanto ao horário de uso, $88,8 \%$ providenciam os medicamentos antes de acabarem, $43,3 \%$ alegam que tiveram problemas referentes aos fármacos e a maioria, durante o período de tratamento, não mudou o medicamento $(74,2 \%)$. Dos hipertensos $7,2 \%$ revelaram que quando se sentem bem com os medicamentos deixam de tomar e $12,4 \%$ quando se sentem mal com os medicamentos interrompem o tratamento.

Com relação aos serviços e aos profissionais da saúde, $69,1 \%$ não faz acompanhamento médico frequentemente, sendo a dificuldade de conseguir consulta o motivo mais encontrado $(42,3 \%)$. 
Tabela 1: Perfil dos pacientes hipertensos atendidos em uma drograria de Porto Alegre, RS

\begin{tabular}{|c|c|c|}
\hline Variáveis & $n^{\circ} 97$ & $\%$ \\
\hline \multicolumn{3}{|l|}{ Escolaridade } \\
\hline Ensino fundamental & 50 & 51,5 \\
\hline Ensino médio & 45 & 46,4 \\
\hline Ensino superior & 2 & 2,1 \\
\hline \multicolumn{3}{|c|}{ Renda familiar em salários minimo } \\
\hline$<2$ & 34 & 35,1 \\
\hline 3 a 4 & 50 & 51,5 \\
\hline 5 a 10 & 13 & 13,4 \\
\hline \multicolumn{3}{|c|}{ Familiares com hipertensão arterial } \\
\hline Sim & 89 & 91,8 \\
\hline Não & 8 & 8,2 \\
\hline \multicolumn{3}{|l|}{ Massa corporal } \\
\hline Peso normal & 14 & 14,4 \\
\hline Sobrepeso & 56 & 57,7 \\
\hline Obesidade & 27 & 27,8 \\
\hline \multicolumn{3}{|l|}{ Ingestão de gorduras } \\
\hline Frequentemente & 8 & 8,2 \\
\hline As vezes & 89 & 91,8 \\
\hline \multicolumn{3}{|l|}{ Ingestão de frutas } \\
\hline Frequentemente & 67 & 69,1 \\
\hline As vezes & 30 & 30,9 \\
\hline \multicolumn{3}{|l|}{ Ingestão de Verduras } \\
\hline Frequentemente & 61 & 62,9 \\
\hline As vezes & 36 & 37,1 \\
\hline \multicolumn{3}{|c|}{ Apresenta restrição de sal } \\
\hline Sim & 42 & 43,3 \\
\hline Não & 55 & 56,7 \\
\hline \multicolumn{3}{|l|}{ Faz atividade física } \\
\hline Sim & 26 & 26,8 \\
\hline Não & 71 & 73,2 \\
\hline \multicolumn{3}{|l|}{ Fuma } \\
\hline Sim & 22 & 22,7 \\
\hline Não & 75 & 77,3 \\
\hline \multicolumn{3}{|l|}{ Ingere bebida alcoólica } \\
\hline Sim & 25 & 25,8 \\
\hline Não & 72 & 74,2 \\
\hline \multicolumn{3}{|c|}{ Já apresentou doença como } \\
\hline AVC & 2 & 2,1 \\
\hline Isquemia & 1 & 1,0 \\
\hline Infarto & 1 & 1,0 \\
\hline Nenhuma & 93 & 95,9 \\
\hline
\end{tabular}

Tabela 2: Uso de serviços de saúde, conhecimento e adesão ao tratamento anti-hipertensivo pelos pacientes hipertensos atendidos em uma drograria de Porto Alegre, RS

\begin{tabular}{l|c|c|}
\hline \multicolumn{1}{|c|}{ Variáveis } & $\mathbf{n}^{\circ} \mathbf{9 7}$ & $\%$ \\
\hline \multicolumn{2}{|c|}{ Esquece de tomar os medicamentos } \\
\hline Sim & 18 & 18,6 \\
\hline Não & 26 & 26,8 \\
\hline Às vezes & 53 & 54,6 \\
\hline Cuidadoso com horários de medicamentos \\
\hline Sim & 44 & 45,4 \\
\hline Não & 26 & 26,8 \\
\hline Às vezes & 27 & 27,8 \\
\hline Interrompe o tratamento quando se sente bem \\
\hline Sim & 7 & 7,2 \\
\hline Não & 55 & 56,7 \\
\hline Às vezes & 35 & 36,1 \\
\hline
\end{tabular}

Interrompe o tratamento quando se sente mal

\begin{tabular}{|l|l|l|}
\hline Sim & 12 & 12,4 \\
\hline Não & 38 & 39,2 \\
\hline Às vezes & 47 & 48,5 \\
\hline
\end{tabular}

\section{Providencia medicamentos antes de acabar}

\begin{tabular}{|l|l|l|}
\hline Sim & 85 & 88,8 \\
\hline Não & 11 & 11.3 \\
\hline
\end{tabular}

Faz acompanhamento médico

\begin{tabular}{|l|l|l|}
\hline Sim & 30 & 30,9 \\
\hline Não & 67 & 69,1 \\
\hline
\end{tabular}

Se não, por quê?

\begin{tabular}{|l|l|l|}
\hline Falta de Tempo & 20 & 20,6 \\
\hline
\end{tabular}

\begin{tabular}{|l|l|l|}
\hline Difícil conseguir consulta & 41 & 42,3 \\
\hline
\end{tabular}

\begin{tabular}{|l|l|l|}
\hline Esquecimento & 6 & 6,2 \\
\hline
\end{tabular}

Quem orienta o tratamento

\begin{tabular}{|l|c|c|}
\hline Médico & 33 & 34,0 \\
\hline Enfermeiro & 1 & 1,0 \\
\hline Farmacêutico & 40 & 41,2 \\
\hline Outros & 23 & 23,7 \\
\hline Tem melhorado com o tratamento & & \\
\hline Sim & 66 & 68,0 \\
\hline Não & 31 & 32,0 \\
\hline Total & 97 & 100,0 \\
\hline
\end{tabular}

Cronicidade da hipertensão arterial

\begin{tabular}{|l|c|c|}
\hline Toda vida & 45 & 46,4 \\
\hline Não sabe & 27 & 27,8 \\
\hline Doença grave & 18 & 18,6 \\
\hline Passageira & 7 & 7,2 \\
\hline Consequência da hipertensão arterial & \\
\hline Consequências graves & 43 & 44,3 \\
\hline Não tenho conhecimento & 43 & 44,3 \\
\hline Consequências leves & 8 & 8,2 \\
\hline Sem consequências & 3 & 3,1 \\
\hline
\end{tabular}


A maioria dos indivíduos não está satisfeita com o atendimento de saúde (76,3\%) e a maior parte dos entrevistados alegou ser questionada pelo médico quanto ao uso de anti-hipertensivos $(55,7 \%)$. Parte dos entrevistados alegou ser orientada pelo farmacêutico a tomar corretamente seus medicamentos $(41,2 \%)$, sendo que $68 \%$ dos hipertensos acreditavam estar melhorando com o tratamento utilizado.

Quanto ao conhecimento dos pacientes em relação à doença se observou que uma elevada parte dos participantes $(44,3 \%)$ não tem conhecimento das consequências da hipertensão e $27,8 \%$ dos entrevistados não sabem qual é a cronicidade da doença; $18,6 \%$ consideram uma doença grave e 7,2\% acham que é uma doença passageira.

Com a análise dos resultados observou-se que mais da metade dos entrevistados $(62,9 \%)$ respondeu que não esclarece suas dúvidas com o médico em relação à hipertensão, como mostrado na Figura 1.

Na Figura 2, pode ser observado que 53,6\% dos pacientes hipertensos encontravam-se com a pressão arterial igual ou maior que $140 \mathrm{mmHg}$ X $90 \mathrm{mmHg}$, na verificação no dia da entrevista. Para a PAD encontrou-se a média de 87,63 com desvio padrão 8,24 e para a PAS a média foi de 140,11 com desvio padrão de 10,03 .

Figura 1: Informação do médico aos pacientes entrevistados quanto às dúvidas sobre hipertensão

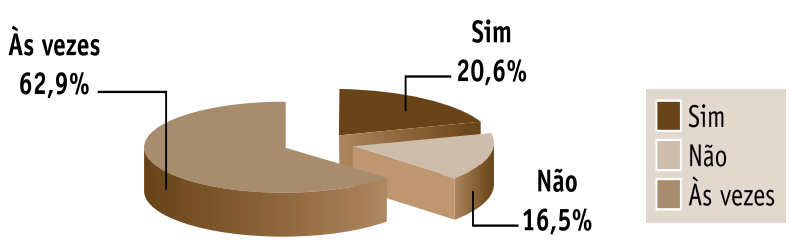

Figura 2: Perfil da pressão arterial dos pacientes hipertensos atendidos em uma drograria de Porto Alegre, RS

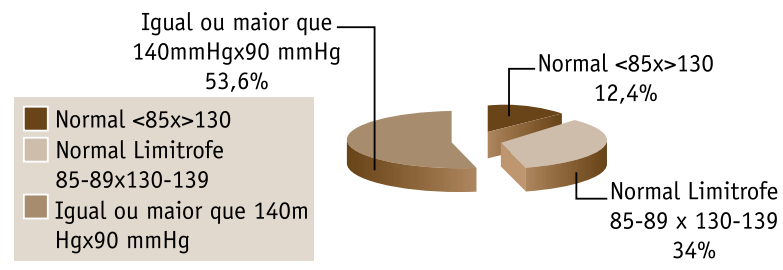

Como pode ser observado na Tabela 3, o tratamento medicamentoso mais utilizado foi a monoterapia $(58,8 \%)$, sendo os fármacos da classe ARAII os de maior escolha (50,9\%). Já para a combinação entre 2 classes de anti-hipertensivos (32\%), a mais prescrita pelos médicos foi diuréticos e IECA (com 61,3\%).

\section{DISCUSSÃO}

Neste estudo observou-se que em relação ao gênero, 58,8\% eram pacientes femininos. Em estudos de base populacional, realizados também em regiões urbanas, foram encontradas prevalências semelhantes às deste estudo. Este fato sugere uma atenção feminina maior nos aspectos de atenção à saúde, já que a procura pelos medicamentos denota um interesse na utilização dos mesmos $(15,16)$. Mais da metade dos pacientes analisados tinha idade superior a 45 anos $(63,9 \%)$, indicando que esta doença é mais prevalente em indivíduos de idade mais elevada. Estes dados são corroborados por outros trabalhos, que também demonstram que a hipertensão arterial é comum em adultos mais velhos. Isto é observado porque podem ocorrer alterações vasculares fisiológicas durante o processo de envelhecimento, que reduzem a distensão arterial, aumentam processos ateroscleróticos e o volume plasmático $(15,17,18)$.

Tabela 3: Perfil de pacientes hipertensos tendidos em drogaria de Porto Alegre, RS: tratamento medicamentoso

\begin{tabular}{|c|c|c|}
\hline Variáveis & $\begin{array}{l}n^{\circ} \\
97\end{array}$ & $\%$ \\
\hline \multicolumn{3}{|l|}{ Monoterapia } \\
\hline Classe medicamentosa & 57 & 58,8 \\
\hline Diuréticos & 4 & 8,8 \\
\hline Inibidores adrenérgicos & 7 & 12,3 \\
\hline Bloqueadores dos canais de cálcio & 2 & 3,5 \\
\hline IECA & 14 & 24,6 \\
\hline ARA II & 29 & 50,9 \\
\hline Total & 57 & 100,0 \\
\hline \multicolumn{3}{|c|}{ Combinação entre 2 classes de anti-hipertensivos } \\
\hline Classe medicamentosa & 31 & 32 \\
\hline Inibidores adrenérgicos + IECA & 4 & 12,9 \\
\hline Diuréticos + IECA & 19 & 61,3 \\
\hline Diuréticos + ARA II & 3 & 9,7 \\
\hline Diuréticos + Inibidores Adrenérgicos & 3 & 9,7 \\
\hline Bloqueadores de Canais de Cálcio + ARA II & 1 & 3,2 \\
\hline Bloqueadores de Canais de Cálcio + IECA & 1 & 3,2 \\
\hline Total & 31 & 100,0 \\
\hline \multicolumn{3}{|c|}{ Combinação entre 3 classes de anti-hipertensivos } \\
\hline Classe medicamentosa & 9 & 9,3 \\
\hline Diuréticos + Inibidores Adrenérgicos +IECA & 5 & 55,6 \\
\hline Diuréticos + Bloqueadores dos Canais de Cálcio + ARA II & 1 & 11,1 \\
\hline Diuréticos + IECA + ARA II & 1 & 11,1 \\
\hline Diuréticos + Bloqueadores dos Canais de Cálcio + IECA & 2 & 22,2 \\
\hline Total & 9 & 100,0 \\
\hline
\end{tabular}


A maior parte dos indivíduos entrevistados ingeria alimentos ricos em gordura (91,8\%); 30 a $40 \%$ deles não ingeriam diariamente verduras e frutas, enquanto $56,7 \%$ não apresentavam restrição ao sal. Estudos comprovam a importância de uma alimentação balanceada tanto em pessoas não hipertensas quanto em indivíduos hipertensos. Mudanças na dieta têm o potencial de prevenir a hipertensão arterial, podendo servir como tratamento prévio à terapia medicamentosa. Entre os hipertensos que já estão em tratamento com fármacos, mudanças dietéticas, incluindo uma redução no consumo de sal, podem vir a complementar o tratamento, reduzindo ainda mais a pressão arterial $(19,20,21)$.

Uma grande parte dos pacientes encontrava-se em sobrepeso $(57,7 \%)$, não fazia atividades físicas $(72,2 \%)$, além de ingerir bebidas alcoólicas e fazer uso de tabaco. Conforme é indicado para o controle da hipertensão, os indivíduos devem adotar um estilo de vida saudável, eliminando hábitos que constituem fatores de risco para a doença. Entretanto, observa-se que é grande a dificuldade para a aquisição de hábitos saudáveis, pois a tomada da decisão, apesar de necessária, constitui uma decisão pessoal. Mudar o estilo de vida é uma tarefa difícil e quase sempre é acompanhada de muita resistência, por isso, a maioria das pessoas não consegue fazer modificações e, especialmente, mantê-las por muito tempo $(20,21,22)$.

Dos pacientes hipertensos, mais da metade encontrava-se com a pressão arterial igual ou maior que $140 \mathrm{mmHg}$ x $90 \mathrm{mmHg}$ na verificação no dia da entrevista. Este aumento pode ter sido observado porque a medição da pressão arterial foi executada somente uma vez durante o estudo, e podem existir inúmeros motivos para elevação da pressão arterial no dia da entrevista, incluindo estresse, síndrome do avental branco e inclusive, má adesão ao tratamento anti-hipertensivo $(22,23,24)$. Porém, esta única medida realizada na drogaria foi aliada ao acompanhamento dos pacientes por seus médicos, que demonstrava que os valores pressóricos não representavam a normotensão (dados não apresentados).

Analisando a adesão ao tratamento medicamentoso, observou-se que $88,8 \%$ dos indivíduos providenciavam os medicamentos antes que os mesmos acabassem, mas mesmo tendo posse da medicação, alguns pacientes alegaram esquecer de tomá-la diariamente e $81,4 \%$ alegaram esquecer algumas vezes. Além disso, muitos dos participantes não são cuidadosos com os horários da medicação, mas apenas $7 \%$ afirmaram que quando se sentem bem com os medicamentos deixam de tomá-los e $35 \%$ responderam que algumas vezes deixam de realizar a administração do medicamento. Quando se sentem mal com o fármaco, $12 \%$ dos pacientes interrompem o tratamento e $47 \%$ alegaram que algumas vezes interrompem o tratamento. Observando estes resultados, é possível observar uma situação de má adesão ao tratamento entre os pacientes do estudo, pois em diferentes situações uma grande parte dos mesmos deixava de tomar os medicamentos. Essa má adesão pode ser devida à falta de informação sobre a doença, pois se observou que os entrevistados alegaram não conseguir esclarecer suas dúvidas em relação à hipertensão e não ter conhecimento das consequências $(44,3 \%)$ e da cronicidade da hipertensão $(27,8 \%)$. Este desconhecimento também pode ser explicado pelo baixo grau de instrução e condição socioeconômica, considerando que apenas $2,1 \%$ possuíam ensino superior, $51,5 \%$ cursaram somente o ensino fundamental, e $86,6 \%$ tinham renda abaixo de 4 salários mínimos.

Em relação aos serviços de saúde, foi verificado que $67 \%$ dos entrevistados não fazem acompanhamento médico frequentemente, sendo a dificuldade de conseguir consulta o motivo principal, descrito por $41 \%$ dos entrevistados. A maioria dos pacientes não está satisfeita com o atendimento de saúde prestado no sistema público de saúde. Conforme relatado por estudos, a adesão pressupõe que um paciente tenha o conhecimento, a motivação, as habilidades e os recursos necessários para acompanhar os cuidados recomendados pelo profissional de saúde. A falta de adesão, pouca ou nenhuma persistência em tomar os medicamentos anti-hipertensivos resulta em hipertensão não controlada $(25,26,27)$.

Neste estudo, o tratamento medicamentoso mais prevalente foi a monoterapia, sendo os fármacos da classe antagonistas do receptor de angiotensina II, os de maior escolha, com $50,9 \%$ da utilização. Isto pode ser devido ao fato de esta classe apresentar um mecanismo de ação semelhante a dos inibidores da enzima conversora de angiotensina, atuando no sistema renina-angiotensina-aldosterona e diferenciando-se por não produzir interferências em outros sistemas hormonais e vias metabólicas iniciais, de modo que não produzem aumento de bradicinina, além de possuírem boa tolerabilidade (28). Já para a combinação entre 2 classes de anti-hipertensivos dos pacientes, a combinação mais prescrita pelos médicos, com $61,3 \%$ da utilização, foi entre diuréticos e inibidores da enzima conversora de angiotensina, uma vez que estes fármacos apresentam mecanismos de ação diferentes e complementares, e um maior efeito protetor sobre órgãos (16). Para as outras combinações de tratamento, a classe mais escolhida foi a dos diuréticos, devido ao baixo custo e também sua eficácia e tolerabilidade (29).

A principal limitação do presente estudo, é que o mesmo faz uma descrição dos indivíduos analisa- 
dos, não havendo a realização de análise inferencial. Este tipo de análise não foi feito porque o objetivo deste trabalho não foi realizar uma análise comparativa entre diferentes grupos, já que a amostra foi limitada a apenas uma drogaria da cidade de Porto Alegre. Novos estudos serão realizados, em drogarias de outros bairros da cidade a fim de se traçar um perfil de comparação entre indivíduos de diferentes regiões de Porto Alegre, levando-se em consideração variações no perfil sócioeconômico dos bairros. Adicionalmente, existem perspectivas de avaliação do impacto das orientações do farmacêutico, pois a hipótese é de que, aderindo corretamente ao tratamento, consequentemente os pacientes terão maiores chances de conseguirem sucesso terapêutico.

\section{CONCLUSÃO}

Com esse trabalho foi possível observar a ocorrência da má adesão dos indivíduos ao tratamento anti-hipertensivo, que pode estar relacionada à falta de informação. Os resultados de baixa adesão observados neste estudo nos possibilitam uma análise crítica e reflexiva sobre a necessidade de intensificar o atendimento farmacêutico desta drogaria, auxiliando estes pacientes na correta utilização dos medicamentos e prestando-lhes informações em relação à HAS, explicando-lhes os benefícios e os riscos da terapia anti-hipertensiva, com a finalidade de conduzi-los a um estilo de vida mais saudável, eliminando ou controlando os riscos da HAS.

\section{REFERÊNCIAS}

1. BRASIL. Ministério da Saúde. Secretaria de Vigilância em Saúde. Secretaria de Gestão Estratégica e Participativa. Vigitel Brasil 2010: vigilância de fatores de risco e proteção para doenças crônicas por inquérito telefônico. Brasília. 2011. Disponível em: http://portal.saude.gov.br/ portal/arquivos/pdf/vigitel_2010_preliminar_web.pdf acesso em: 18/10/2011.

2. Tsai WC. Treatment options for hypertension in high-risk patients. Vasc Health Risk Manag. 2011; 7 (1): 137-141.

3. Cohuet G, Struijker BH. Mechanisms of target organ damage caused by hypertension: Therapeutic potential. Pharmacol e Ther. 2006; 111: 81-98.

4. Galzerano D, Capogrosso C, Di Michele S, Bobbio E, Paparello $\mathrm{P}$, Gaudio C. Do we need more than just powerful blood pressure reductions? New paradigms in end-organ protection. Vasc Health Risk Manag. 2010; 6: 479-494.

5. Romana O, Badillas M, Dussaubata AM. La hipertensión arterial, ¿Sólo un biomarcador de la enfermedad ateroesclerótica sistémica? Rev Med Chile. 2010; 138: 346-351.

6. Hamm LL, Hering-Smith KS. Pivotal Role of the Kidney in Hypertension. Am J Med Sci. New Orleans. 2010; 340 (1): 30-32.

7. Grossman MDE. Blood Pressure: The Lower, the Better. Diabetes Care. 2011; 34 (2): 308-312.

8. Souza MG, Pimenta ES, Borelli FAO. Interações e Associações Medicamentosas no Tratamento da Hipertensão - Combinações Fixas. Rev Bras Hipertens. 2009; 16 (4): 237-241.

9. Busnello RG, Melchior R, Faccin C, Vettori D, Petter J, Moreira LB, Fuchs FD. Características Associadas ao Abandono do Acompanhamento de Pacientes Hipertensos Atendidos em um Ambulatório de Referência. Arq Bras de Cardiol. 2001; 76 (5): $349-51$.

10. Santos ZMSA, Frota MA, Cruz DM, Holanda SDO. Adesão do Cliente Hipertenso ao Tratamento: Análise com Abordagem Interdisciplinar. Texto Contexto Enferm. 2005; 14 (3): 332-40.
11. Jesus ES, Augusto MAO, Gusmão J, Mion Jr D, Ortega K, Pierin AMG. Perfil de um Grupo de Hipertensos: Aspectos Biossociais, Conhecimentos e Adesão ao Tratamento. Acta Paul de Enferm, São Paulo, 2008; 21 (1): 59-65.

12. Souza VV, Bertoncin ALF. Atenção Farmacêutica para Pacientes Hipertensos - Nova Metodologia e a Importância dessa Prática no Acompanhamento Domiciliar. Rev Bras Promoção da Saúde. 2008; 21 (3): 224-230.

13. Mousinho LPM, Moura MES. Hipertensão Arterial: Fatores Relacionados à Adesão do Cliente com Hipertensão ao Tratamento Medicamentoso. Saúde Coletiva. 2008; 5: 212-216.

14. Rosário TM, Scala LCN, França GVA, Pereira MRG, Jardim PCBV. Prevalência, Controle e Tratamento da Hipertensão Arterial Sistêmica em Nobres - MT. Arq Bras Cardiol. 2009; 93 (6): 672-678.

15. Kramoh EK, N'goran INK, Aké-Traboulsi E, Anzouan-Kacou JB, Konin CK, Coulibaly I, Traoré F, Agbechi YM, Guikahue MK. Hypertension management in an outpatient clinic at the Institute of Cardiology of Abidjan (Ivory Coast). Arch Cardiovasc Dis. $2011 ; 104,558-564$.

16. Iyer AS, Ahmed MI, Filippatos GS, Ekundayo OJ, Aban IB, Love TE, Nanda NC, Bakris GL, Fonarow GC, Ahmed A. Uncontrolled hypertension and increased risk for incident heart failure in older adults with hypertension: findings from a propensity-matched prospective population. J Am Soc Hypertens. 2010; 4(1): 22-31.

17. Dennis T, Meera NK, Binny K. Medication adherence and associated barriers in hypertension management in India. CVD Prevent Control. 2011; 6: 9-13.

18. Appel LJ, Brands MW, Daniels AR, Karanja N, Elmer PJ, Sacks FM. Dietary Approaches to Prevent and Treat Hypertension: A Scientific Statement From the American Heart Association. Hypertension. 2006; 47: 296-308.

19. Brito DMS, Araújo TL, Galvão MTG, Moreira TMM, Lopes, MVO. Qualidade de vida e percepção da doença entre portadores de hipertensão arterial. Cad Saúde Pública. 2008; 24(4): 933-940. 
20. Santos ZMSA, Lima HE. Tecnologia educativa em Saúde na Prevenção da Hipertensão Arterial em Trabalhadores: Análise das mudanças no Estilo de Vida. Texto Contexto Enferm, 2008; 17(1): 90-7.

21. Bosworth HB, Olsen MK, Neary A, Orr M, Grubber J, Svetkey L, Adams M, Oddone EZ. Take Control of Your Blood pressure (TCYB) study: A multifactorial tailored behavioral and educational intervention for achieving blood pressure control. Patient Educ Couns. 2008; 70: 338-347.

22. Efstratopoulos AD, et al. Prevalence, Awareness, Treatment and Control of Hypertension in Hellas, Greece. Am J Hypertens. 2006; 19: 53-60.

23. Rothwell PM. Limitations of the usual blood-pressure hypothesis and importance of variability, instability, and episodic hypertension. Lancet. 2010; 375: 938-48.
24. Hill MN, Miller NH, Degeest S; American Society of Hypertension Writing Group, Materson BJ, Black HR, Izzo JL Jr, Oparil $\mathrm{S}$, Weber MA. Adherence and persistence with taking medication to control high blood pressure. J Am Soc Hypertens. 2011; 5(1): $56-63$.

25. Fung V, Huang J, Brand R, Newhouse JP, Hsu J. Hypertension Treatment in a Medicare Population: Adherence and Systolic Blood Pressure Control. Clin Ther. 2001; 29: 5.

26. Krousel-Wood MA, Muntner P, Islam T, Morisky DE, Weber LS. Barriers to and Determinants of Medication Adherence in Hyper tension Management: Perspective of the Cohor t Study of Medication Adherence Among Older Adults. Med Clin North Am. 2009; 93: 753-169.

27. Armario P, Waeber B. Therapeutic strategies to improve control of hypertension. J Hypertens. 2013 Mar; 31 Suppl 1:S9-12. 